\title{
POINT SETS IN THREE AND HIGHER DIMENSIONS AND THEIR INVESTIGATION BY MEANS OF A UNIFIED ANALYSIS SITUS*
}

\author{
BY R. L. WILDER
}

1. Introduction. When I was invited to give this symposium lecture, I hesitated somewhat to speak upon a field of mathematics which has already been represented before this Society by three Colloquia $\nmid$ and several lectures. $\ddagger$ It occurred to me, however, that each of these lectures and Colloquia had been devoted to one of the two special "branches" of analysis situs, that is, either to combinatorial topology or to set-theoretic topology. $\S$ ( $I$ think it is fair to state that most of the workers in analysis situs can be identified, by the evidence of their published works, with one of these "branches.") We have had no report before this Society which makes clear the relations between these schools of analysis situs-why there are two schools and what is the difference between them-nor is it evident that any but a few topologists are aware of the tendency, which has become manifest within the past few years, for the lines of demarcation between these "branches" of analysis situs to disappear. There are reasons, indeed, for believing that many topologists do not approve of this tendency, whether for esthetic reasons or because of their faith in the power of their own

* Symposium Lecture delivered at the meeting of the Society at Chicago, April 8, 1932.

$\dagger$ O. Veblen, Analysis Situs, Colloquium Publications, vol. 5, Part II; R. L. Moore, Foundations of Point Set Theory, Colloquium Publications, vol. 13; S. Lefschetz, Topology, Colloquium Publications, vol. 12.

$\ddagger \mathrm{R}$. L. Moore, Report on continuous curves from the viewpoint of analysis situs, this Bulletin, vol. 29 (1923), pp. 289-302; J. R. Kline, Separation theorems and their relation to recent developments in analysis situs, ibid., vol. 34 (1928), pp. 155-192; E. W. Chittenden, On the metrization problem and related problems in the theory of abstract sets, ibid., vol. 33 (1927), pp. 13-34; T. H. Hildebrandt, The Borel theorem and its generalizations, ibid., vol. 32 (1926), pp. 423-474.

$\S$ It might seem that in the case of Lefschetz' Colloquium, this remark is not true, since he considers topics that are ordinarily considered as part of the set-theoretic topology, for example, compact metric spaces. However, as we shall show, it is in method, not subject matter, that the two schools of topology differ, and in this sense Lefschetz' book is combinatorial. 
methods, I do not know. However, such a tendency exists, particularly in the investigation of higher dimensional point sets, as well as in the study of compact metric spaces, and, in my opinion, has justified itself in the solution of problems which heretofore had defied the efforts of either the set-theoretic or the combinatorial methods, as well as in the providing of new and simpler solutions of older problems.

I hope I am justified, then, in what I propose to do, namely: (1) To maintain the thesis that there is only one branch of mathematics to be known as analysis situs, or topology, and that what are known as set-theoretic topology and combinatorial topology are really two methods within topology having the same aim. (2) To report upon the progress that has been made in the investigation of point sets in higher dimensions, and to demonstrate the tendency of the set-theoretic and combinatorial methods to unite in this investigation.

In carrying out these purposes, I shall divide my lecture into two parts. In the first part I shall give a brief introduction to the set-theoretic and combinatorial methods, trying to make clear what they are and what distinguishes them from one another, as well as what they can and what they apparently cannot accomplish separately.

In the second part, I propose to report on what has been accomplished in the topology of point sets in $E_{n}(n \geqq 3)$, with a view not only to furnish a summary of the present-day status of this study, but to show how the set-theoretic and combinatorial methods unite in this study to form what we might call a unified analysis situs.

Before concluding these introductory remarks, I wish to quote from two of the above-mentioned lectures. In concluding his lecture at Kansas on continuous curves from the viewpoint of analysis situs," ${ }^{*}$ R. L. Moore stated: "Beyond certain propositions which hold true for both two and three dimensions, comparatively little is known concerning continuous curves in threedimensional space. A fruitful field for future investigation is afforded in that branch of analysis situs which has to do with various special types of continuous curves such as simple continuous arcs, simple closed curves, and simple closed surfaces, in space of three dimensions. Little is known concerning the

* Loc. cit. 
relation of such point sets to their complementary domains." The propositions which Moore referred to here as holding for both two and three dimensions, were, in the main, propositions concerning the internal properties of continuous curves which are now recognized to hold in general metric spaces, and have not to do with the relation of continuous curves, or, as I shall call them, Jordan continua, to their complementary domains. As to the relations of arcs, simple closed curves, and surfaces to their complementary domains, most of the fundamental problems concerning these types of Jordan continua have been cleared up since the date of Moore's lecture, as we shall see below. Furthermore, we are in possession of many details concerning the relations of general closed point sets to their complements in $E_{n}$, with which we were unfamiliar at the time of Moore's report, and we shall see that many of these relations, of necessity, take a combinatorial form such as is exhibited in the duality relations.

As recently as $1927, \mathrm{~J}$. R. Kline stated in his concluding remarks of a lecture on separation theorems in analysis situs, ${ }^{*}$ "As is evident from the matter presented in this paper, our knowledge of separation properties of sets immersed in three or more dimensions is comparatively limited. Here is an extremely interesting and important field that is practically untouched." We shall see that a great deal has since been accomplished in regard to these separation theorems in addition to the results which Kline mentions; as a matter of fact, we have a solution, by a unified analysis situs, of two of the main problems to which Kline refers in his lecture. $\dagger$

In view of these remarks of Moore and Kline as to the general lack of knowledge concerning higher dimensional point sets, I think it may be particularly fitting to report upon the knowledge which we have recently gained-a knowledge which I believe is now considerable. We shall see, moreover, that many problems which not long ago seemed far beyond our scope, are now, thanks to a unified analysis situs, well within our grasp.

* Loc. cit.

$\dagger$ That is, the converse of the Jordan-Brouwer separation theorem in $E_{3}$ (see Kline, loc. cit., pp. 158-159), and the finding of necessary and sufficient conditions that the sum of two closed connected sets, neither of which separates $E_{3}$, may have a sum that separates $E_{3}$ (see Kline, loc. cit., p. 191, problem (b)). 
2. Definition of Analysis Situs. In a paper which recognizes frankly the existence of two "schools" of analysis situs, we should first make clear just what analysis situs is. Despite the attention which this field has received from this Society, I am frequently assailed by the conviction that many of us do not know what analysis situs is, nor what it is trying to do. Some of the difficulty is due to the name itself, since the word "analysis" has, for most mathematicians, a very different connotation from what is intended here. We do not, as a rule, think of geometry when we hear the word "analysis." Yet analysis situs is geometry, differing from other branches of geometry only in the type of transformation which characterizes it, and in the great generality of the spaces which it often considers.

Perhaps this difficulty is a modern one, since the effect of Klein's Erlanger Programm seems to have clarified, in the mathematical mind of the past century, the distinction between that type of geometry which it called, variously, "topology," "geometria situs," "analysis situs," and other types of geometry. A recent speaker* before the Deutsche Mathematiker-Vereinigung suggests that Listing adopted the term "Topologie" in order to avoid a possible confusion with the "Geometrie der Lage" of von Staudt. Perhaps we should use the term "topology" today in preference to "analysis situs," in order to suggest things geometrical.

As a branch of geometry, analysis situs has distinguished itself by breaking away from the bounds that held other geometries to special types of spaces. The limit point or neighborhood notion led (Fréchet, Riesz, Hausdorff) to the concept of general topological or abstract spaces, a concept which may be said to form the foundation of modern topology. In terms of neighborhood, a point $P$ is said to be a limit point of a point set $M$ if every neighborhood of $P$ contains at least one point of $M$ distinct from $P$. Then two point sets $A$ and $B$ are said to be homeomorphic if there exists a point for point correspondence between $A$ and $B$ which preserves limit points; that is, a correspondence such that if $M$ is a point set and $P$ a point which lies in $A$.

* G. Feigl, Geschichtliche Entwickelung der Topologie, Jahresbericht der Deutschen Mathematiker-Vereinigung, vol. 37 (1928), pp. 273-286. 
and $M$ and $P^{\prime}$ are their correspondents, respectively, in $B$, then $P$ is a limit point of $M$ if and only if $P^{\prime}$ is a limit point of $M^{\prime}$. This relation between $A$ and $B$ is also of ten expressed by saying that there exists a topological transformation of $A$ into $B$ (or $B$ into $A$ ). Analysis situs, or topology, is that branch of geometry whose objects of study are the properties of point sets that are invariant under topological transformations.

Why do we have two "branches," or "schools," of analysis situs? If we were to glance at some of the literature on topology we might be led to conclude, judging from the differences in the problems studied, that there must be two aims or goals in analysis situs which lead to different types of problems. This is not the case. If one has a mathematical tool which will serve to attack a certain type of problem, he is very likely to seek that type of problem-no doubt this accounts for some of the literature on analysis situs. There is, in fact, only one goal in analysis situs, and this is indicated in the above definition, and this actual ultimate goal should not be crowded from our sight by what we seek as our immediate goals.

We shall find the answer to the question just proposed in the works of Cantor and his followers, and in the works of Riemann and Poincaré. Due to these, there have evolved two pathways to the ultimate goal, which we may call methods, and we shall see that it is of the set-theoretic method, instead of the settheoretic branch or school that we should speak, and the same applies to the combinatorial method. First, however, let us view the fundamentals of these two methods.

3. The Set-Theoretic Method. The set-theoretic method is based directly on the neighborhood-limit point notion, and, in general, does not assume any such structural basis as the homeomorph of the fundamental cube of $E_{n}$. The intuitive notion of connectedness in geometric forms is given a precise formulation as follows. A set of points $M$ is called connected if it is not the sum of mutually exclusive non-vacuous sets $M_{1}$ and $M_{2}$, neither of which contains a limit point of the other.* Regarding the properties of sets which are known to be connected, but about which we have no additional information, some things of im-

* This is the so-called Lennes-Hausdorff definition. 
portance have been found,* but the theory at present in this regard is not very extensive, and to date does not yield to the combinatorial method at all. However, if we further restrict a set by requiring that it be closed, that is, that it contain all of its limit points, then we get what is known as the continuum, and a more extensive theory. Most of the early set-theoretic work in topology was done on continua, although there exists an extensive theory of sets which are closed, but not necessarily connected. Among the continua that have received considerable attention are the Jordan continua. Although characterized analytically by Jordan, they may be characterized by topological properties as follows. A set $M$ is locally connected if, given any point $P$ of $M$ and a neighborhood $U$ of $P$, there exists a neighborhood $V$ of $P$ contained in $U$ such that all points of $M$ in $V$ lie in a connected subset of $M$ which itself lies in $U$. A simple example of a set that is not locally connected is the set $M$ in the plane consisting of (1) all points on the curve $y=\sin 1 / x$ for $0<x \leqq 1$ and (2) all points $(x, y)$ such that $x=0$ and $-1 \leqq y \leqq 1$. Using as neighborhoods the interiors of circles, it is easily seen that $M$ fails to be locally connected at all points where $x=0$. If, then, we require of a continuum that it be locally connected, we get a generalized Jordan continuum; the addition of the condition that it be compact, that is, that every infinite set in it have a limit point, $\dagger$ yields what is ordinarily known as the Jordan continuum. Most of the simple forms of continua, as the arc, the simple closed curve, the simple surfaces, etc., are Jordan continua; all of the euclidean $n$-dimensional spherical spaces are Jordan continua, and all of the euclidean spaces are generalized Jordan continua. The theory of Jordan continua is very extensive and has been developed almost exclusively by the set-theoretic method. The theory of Jordan continua that lie in the plane has been almost exhaustively developed, both with regard to the internal properties of the continua and as regards their external properties.

Because of their importance, these terms deserve further explanation. By the internal properties of a set, we shall mean

* See, for instance, B. Knaster and C. Kuratowski, Sur les ensembles connexes, Fundamenta Mathematicae, vol. 2 (1921), pp. 206-255.

$\dagger$ In euclidean space this means simply that the continuum lies wholly within some sphere, that is, is bounded. 
those properties which can be described by considering the set itself as the space; if it is a subset of another space, a neighborhood is defined as merely the set of all its points that lie in a neighborhood of the space in which it is imbedded. By external properties of a set we shall mean those properties which have to do with the relations of the set to the space in which it is imbedded; without this imbedding space no external properties exist. Thus, in the plane, if a Jordan continuum is the boundary of a domain, then all its points are accessible from this domain, that is, an optional point $P$ of the continuum is joined to an optional point $Q$ of the domain by an arc which has only $P$ in common with the continuum. Another example: If a Jordan continuum $M$ separates two points $P$ and $Q$ of the plane, then these points are separated by a simple closed curve of $M$. (As we shall see later, this is a special case of a general linking theorem.) Both of the theorems just stated are theorems concerning the external properties of a set. On the other hand, the theorem that if $P$ and $Q$ are points of a Jordan continuum $M$, then there exists an $\operatorname{arc}$ in $M$ with the end points $P$ and $Q$, is a statement concerning the internal properties of the Jordan continuum. Most of the theorems concerning internal properties of Jordan continua extend without any other change than that of terminology to higher dimensional euclidean spaces and to more general spaces. However, in the investigation of the external properties of point sets, particularly those that we shall call properties in the large, the set-theoretic method seems to have been lacking, and this is not surprising owing to its foundation in the neighborhood idea.

In closing this brief description of the set-theoretic method, I think that it will be highly appropriate if I quote from the introduction of Schoenflies' Die Entwickelung der Lehre von den Punktmannigfaltigkeiten, an Ergänzungsband of the Jahresbericht der Deutschen Mathematiker-Vereinigung, published in 1908. A great deal of the set-theoretic work that has been done in this country was inspired by this work of Schoenflies. To quote: "No one will fail to recognize the immense usefulness which the arithmetic tendencies in the domain of geometry have achieved; the set-theoretic method is itself in a certain sense an offspring of arithmetization. Its ideas and methods have all the precision and rigor that characterize the arithmetic treat- 
ment. But the demand to use in geometry exclusively analytical language and ideas would overshoot the goal. Moreover it entails the danger of one's forgetting to investigate more closely the spatial and formlike significance of the analytic symbols and therefore succeeding only to a partial knowledge.

"In fact, this is the development which we encounter on many occasions. We possess, to be sure, a very pure analytic, but a much less pure and precise geometric language . . . .

"In the following report one can easily designate two main groups of mathematical theorems . . . A first group is formed by the general theorems on point sets; they represent the settheoretic and accordingly the arithmetic foundation. A second group is formed by the simple theorems on straight lines, polygons, and polyhedrals, which I assume as given without a closer axiomatic analysis. In these is the conception of form, the intuitively accessible foundation, contained."

What are we to gather from these words of Schoenflies? In the first place he says clearly that in order to preserve a contact with geometry, and with intuitive conceptions of form, it is necessary to use, besides the set-theoretic method, theorems on polygons and polyhedrals. This he does, and since his work is almost exclusively laid in the plane, the properties of polygons are sufficient for his needs. However, at the end of Chapter 5 of this same work, a chapter in which he discusses topological invariants, and proves such theorems as the Jordan Curve Theorem, he says "I have chosen the methods of proof in this chapter in such a way that they permit an extension to configurations in space. Nevertheless the proofs are directly applicable only in a certain part. First, in three-space must be taken into consideration the contrast between curve and surface, and secondly, the connectivity number (referring here to the Riemann connectivity numbers) plays an important role in the theorems and proofs. The connectivity number is an invariant of topology, but without a knowledge of it only a part of the developments of this chapter can be directly extended. Yet one will not consider this a defect in the methods used. For the consideration of the connectivity number in three-space is unavoidable; every method which ignores it would yield only a part of that which is to be proved."*

* See also F. Hausdorff, Grundzüge der Mengenlehre, 1914, p. 335, last paragraph of $\$ 10$. 
Although many of Schoenflies' ideas concerning the nature of sets of points in three-space may look peculiar in the light of our more advanced knowledge, I believe that we may well give thought to the words which I have quoted. Instead of saying today, as Schoenflies said twenty-four years ago, "We possess a very pure analytic, but a much less pure and precise geometric language," we might well say, "We possess a pure set-theoretic language and a precise and rigorous theory of the connectivity of higher dimensional polyhedrals. In order to extend the theorems of the plane to higher dimensions, it is necessary that we combine these two in our investigations." I am confident that this follows the spirit of Schoenflies' ideas. In general, however, the set-theoretic investigators have followed the path indicated by the researches of Schoenflies in the theory of sets of points, but have not retained his general method, nor heeded his suggestions relative to extending his results to three-space.

I have given some idea of the set-theoretic method, which proceeds from the notion of limit point and neighborhood. The theory of the connectivity numbers of higher dimensional polyhedrals, or complexes as we shall call them, has been developed hy the combinatorial method, to which I shall now turn.

4. The Combinatorial Method. There are, broadly speaking, two kinds of combinatorial topology, the pure and the geometric. Although, in dealing with internal properties of sets of points, we shall use chiefly the pure combinatorial topology, it is probably better that we consider the combinatorial method from the standpoint of the geometric type, since it is the more intuitive and less abstract and of great use in the investigation of external properties; formally, however, the difference is superficial.

In the geometric type of combinatorial topology the element with which we operate is the $n$-cell, which for our purposes* we consider as a point set which is homeomorphic with the $n$ dimensional tetrahedron. The boundary cells of the $n$-cell are those $i$-cells $(0 \leqq i \leqq n-1)$ that are the homeomorphs of the $i$-faces of the tetrahedron. For the particular cases $n=0$ and $n=1$, we have, respectively, the point (no boundary cells) and the arc (boundary cells are two 0 -cells). For $n=2$, we have

* As a rule, the $n$-cell is defined to be the homeomorph of the interior of the tetrahedron. 
the homeomorph of the plane triangle together with its interior (boundary cells are three 1-cells and three 0 -cells). An $n$ complex is a configuration consisting of a collection of $n$-cells, called fundamental cells of the complex, where two cells of the collection either have no point in common, or have only a boundary cell of each in common. According to how we define the boundary of a complex we get various types of theories.* Formally speaking, if $K^{n}$ is an $n$-complex, we may represent each $(n-1)$-cell, which is a boundary cell of at least one fundamental cell of $K^{n}$, by a symbol, and denote the boundary of each fundamental cell of $K^{n}$ by a certain algebraic expression in these symbols; then the boundary of $K^{n}$ is, say, the association of the algebraic sum of these expressions with the cells whose symbols occur in it. In the modulo 2 analysis situs, the boundary of the $n$-cell is denoted by the linear expression of the symbols for the boundary $(n-1)$-cells with coefficients unity; the algebraic sums are taken modulo 2 , and thus the boundary of $K^{n}$ is the set of those $(n-1)$-cells that are boundary cells of an odd number of the fundamental cells. If the sum, modulo 2 , of the boundaries of the fundamental cells of $K^{n}$ is zero, that is, if each $(n-1)$-cell is on the boundary of an even number of the fundamental cells, then $K^{n}$ is an $n$-cycle, modulo $2 . \dagger$ If no proper subset of the fundamental cells of an $n$-cycle $K^{n}$ forms an $n$ cycle, and also every point of $K^{n}$ is in a neighborhood (in the set-theoretic sense) of the complex which with its boundary (set-theoretic) is an $n$-cell, then $K^{n}$ is an $n$-manifold, modulo 2 .

Although, in the classical combinatorial topology, orientation of cells is introduced, and Alexander has introduced a modulo $m$ topology, we may limit ourselves for purposes of introduction to the modulo 2 type; particularly since we intend no complete survey of combinatorial topology, but rather propose to indicate only the general method.

The simple closed curve when subdivided into 1-cells so as to form a complex, is a 1-manifold, the only one, in fact. Among the 2-manifolds are the 2 -sphere, or the simple closed surface as the set-theorists call it; also the torus, and, in general, the simple closed surface with any number of handles attached. We notice,

* See Lefschetz, Topology, Chap. I, §14.

$\dagger$ In particular, the boundary of an $n$-cell is an $(n-1)$-cycle, indeed, an $(n-1)$-sphere. A 0 -cycle is any even number of 0 -cells. 
on the torus, that there can be drawn two types of simple closed curves neither of which is the boundary of any collection of the 2 -cells (however these be represented) that make up the torus. This is a topologically invariant property of the torus, and we express it by saying that the Betti number of the torus of dimension 1, or simply the 1-dim. Betti number, is 2 . The 1-dim. Betti number of the sphere is zero. This brings us to the notion of a homology. On a complex $K$ two $i$-cycles are said to be homologous to one another if together they bound some $(i+1)$ complex of $K$. If we denote the $i$-cycles by $C_{1}{ }^{i}$ and $C_{2}{ }^{i}$, we express symbolically the fact just stated as follows:

$$
\left.C_{1}^{i} \sim C_{2}^{i} \quad \text { (modulo } 2, K\right) .
$$

If an $i$-cycle $C^{i}$ of $K$ itself bounds some $(i+1)$-complex of $K$, we write

$$
C^{i} \sim 0
$$

(modulo $2, K)$,

to be read " $C^{i}$ is homologous to zero on $K$, modulo 2." On the 2 -sphere, if $i=1$, (2) holds for every $C^{i}$. As we have seen above, this is not true on the torus. Let us pick out two simple closed curves on the torus of the types mentioned above as not bounding on the torus. Denote them by $C_{1}^{1}$ and $C_{2}^{1}$. Then if $C^{1}$ be another 1-cycle on the torus, we shall find that there exists a sum $C^{1}+\eta_{1} C_{1}{ }^{1}+\eta_{2} C_{2}{ }^{1}$, ${ }^{*}$ where $\eta_{1}$ and $\eta_{2}$ are either 1 or 0 , which bounds some 2-complex on the torus. As we can express this fact by a relation of type 2 , we say that the three 1 -cycles are not linearly independent with respect to homologies on the torus.

We are now ready to generalize further the notions just introduced. On a complex $K$ let $C_{1}{ }^{i}, C_{2}{ }^{i}, \cdots, C_{k}^{i}$ be distinct $i$-cycles having the following properties: (1) there exists no relation of the form $\eta_{1} C_{1}{ }^{i}+\eta_{2} C_{2}{ }^{i}+\cdots+\eta_{k} C_{k}{ }^{i} \sim 0$ (modulo $2, K$ ) unless the $\eta$ 's are all zero, that is, these $i$-cycles are linearly independent with respect to homologies on $K$; (2) if $C^{i}$ is any $i$-cycle of $K$, then either it is one of the cycles of the above set, or it satisfies a relation of the form $C^{i} \sim \eta_{1} C_{1}{ }^{i}+\eta_{2} C_{2}{ }^{i}+\cdots+\eta_{k} C_{k}{ }^{i}$

* All sums are modulo 2 ; thus, the sum of a number of $n$-complexes is the collection of those $n$-cells (and their boundaries) that are in an odd number of the complexes. It is easy to see that the sum of a finite number of $n$-cycles is itself an $n$-cycle (or vacuous); also, the boundary of an $n$-complex is an $(n-1)$ cycle (or vacuous). 
(modulo $2, K$ ), where the $\eta$ 's are either 1 or 0 . Then the above set of $i$-cycles is said to form a basis for the $i$-cycles of $K$ or $i$-basis of $K$, and their number, $k$, is called the $i$-dim. (or $i$ th) Betti number of $K$. Symbolically expressed, this fact is written $p^{i}(K)=k$.

We can also define Betti numbers for the complement of a point set in $E_{n}$. For purposes of simplification, as well as of symmetry, let us consider the $n$-dimensional spherical space $H_{n}$, that is, the set of points $x_{1}^{2}+x_{2}^{2}+\cdots+x_{n+1}^{2}=1$ in $E_{n+1}$. By an easily defined procedure of cutting up (by passing $n$ planes through $H_{n}{ }^{*}$ ), $H_{n}$ may be subdivided into a finite number of $n$-cells as small as we please, whose sum makes up $H_{n}$ and in terms of which $H_{n}$ is an $n$-conplex. Let us adopt once and for all a set $S$ of subdivisions of $H_{n}$, namely, $S_{1}, S_{2}, S_{3}, \cdots$, such that for each $i, S_{i+1}$ is a subdivision of $S_{i}$ and such that if $e$ is any positive number, there is an $i$ for which the cells of $S_{i}$ are all of diameter less than $e$. Let $M$ be any closed set of points in $H_{n}$. If $C^{j}$ is a $j$-cycle of $S_{i}$ which lies wholly in $H_{n}-M$, then $C^{j}$ is said to be homologous to any cycle of $S_{i+h}(h>0)$ which is a mere subdivision of $C^{j}$; and if in any $S_{i+h}$ there exists a complex $K^{j+1}$ whose cells lie wholly in $H_{n}-M$ and whose boundary is $C^{j}$ (or $C^{j}$ subdivided into finer cells), then $C^{j}$ is said to be homologous to zero in $H_{n}-M$; that is, $C^{j} \sim 0,\left(H_{n}-M\right)$. If no such complex $K^{j+1}$ exists in any $S_{i+h}$, then $C^{j}$ is said to be unbounding in $H_{n}-M$, or to link $M$ in $H_{n}$. The definitions of homologies between cycles in $H_{n}-M$, and of linear independence (in $H_{n}-M$ ) between cycles with respect to homologies, are given as before, keeping in mind that the complexes considered must at all times lie in $H_{n}-M$. If we now denote by $p_{i}{ }^{j}\left(H_{n}-M\right)$ the maximum number of independent (in $H_{n}-M$ ) $j$-cycles of $S_{i}$, then the number $p^{j}\left(H_{n}-M\right)=\operatorname{Lim}_{i \rightarrow \infty} p_{i}{ }^{i}\left(H_{n}-M\right)$, which may be finite or infinite, is called the $j$ th Betti number of $H_{n}-M$. This number is invariant for all possible choices of the sequence $S$, as well as for all topological transformations of $M$ in $H_{n}$. In particular, the number $p^{0}\left(H_{n}-M\right)+1$ represents the number of domains complementary to $M$, and as a consequence of the theorem just stated, the number of domains complementary to a closed set in $H_{n}$ (and in $E_{n}$ for $M$ bounded) is an

* See J. W. Alexander, A proof and extension of the Jordan-Brouwer separation theorem, Transactions of this Society, vol. 23 (1922), pp. 333-349. 
invariant external property of $M$. The proof of this theorem is one of the achievements of unified analysis situs.

The case where $M$, instead of being any closed set, is a complex, in the sense previously defined (that is, not necessarily formed from cells of the $S_{i}$ 's), is not only interesting but important for what follows. Connecting the Betti numbers of $M$ and of $H_{n}-M$, we have the fundamental Alexander Duality Theorem :*

$$
p^{i}(M)=p^{n-i-1}\left(H_{n}-M\right), \quad(0 \leqq i \leqq n-1) .
$$

Thus, where the Betti numbers of $M$ are known, we know the Betti numbers of $H_{n}-M$, and conversely. A special case of this theorem is that (chief) part of the classical Jordan-Brouwer separation theorem, proved by Jordan and others for the plane (Jordan Curve Theorem) and by Brouwer for the general case, which states that a topological $(n-1)$-sphere in $H_{n}$ separates $H_{n}$ into just two connected domains; to get the latter theorem we let $i=n-1$ in (3), the Betti number $p^{n-1}(M)$ being equal to 1 since $M$ is the only (unbounding) $(n-1)$-cycle in itself. For the cases where $M$ is a topological $i$-sphere in $H_{n}, p^{i}(M)=1$, and $p^{s}(M)=0$ where $s \neq i$, and there is therefore just one cycle in $H_{n}-M$ that does not bound in $H_{n}-M$, this cycle being of dimensionality $n-i-1$; thus the $i$-sphere is linked by just one independent cycle.

It has been shown independently by Pontrjagin and Frankl that (where $M$ is a complex) the $i$-basis of $M$ and the $(n-i-1)$ basis of $H_{n}-M$ may be so chosen that the cycles of the two bases link uniquely; that is, so that a cycle of the $i$-basis of $M$ links one and only one cycle of the $(n-i-1)$-basis of $H_{n}-M$, and conversely.

In the pure combinatorial method, instead of dealing with the $n$-cell as the homeomorph of the $n$-dimensional tetrahedron, we consider any set of $n+1$ points as forming the $n$-cell. The ${ }_{n} C_{i+1}$ sets of $i+1$ points of such a set form the $i$-cells that enter into the boundary of the $n$-cell. The definitions of complex, cycle, etc., are given as before. The pure combinatorial method is of particular value in studying the topological properties of general closed point sets. Because of the foundation upon finite sets

* See J. W. Alexander, loc. cit. 
of points its application is not restricted to subsets of euclidean spaces.

5. Contrast of the Two Methods. I have tried to indicate the general nature of the set-theoretic and combinatorial methods, the one based on the neighborhood-limit point notion, the other based essentially on the euclidean tetrahedron. If every topologist used both of these methods, there would be hardly any raison d'être for the present report. As I have already stated, however, topologists in general are either set-theoretic or combinatorial; the consequences of this situation may as well be frankly stated: a too prevalent tendency on the part of one to ignore the works of the other; the inability to extend many results of the set-theoretic topology to higher dimensions; overlapping of results; and inexcusable delay in the discovery of important general theorems.

Why is it necessary, as Schoenflies seems to have recognized, to use both methods in the investigation of point sets in higher dimensions? The answer, I believe, lies in the difference between what we may call local properties, and im grossen properties or properties in the large. We may say that a local property, as distinguished from a property in the large, is a property that concerns the arbitrarily small neighborhoods of a point.

Glance at the literature on set-theoretic topology and notice the preponderance of local properties. The property of local connectedness is basic in the theory of Jordan continua. The Menger-Urysohn theory of dimension is based on a local property. On the other hand the combinatorial method deals essentially with properties in the large. As an example, the theory of linking, which occupies a central position in combinatorial topology, is based upon a property in the large.

To be sure, either of the above methods can apparently be extended so as to take care of both types of properties in many cases. The combinatorial method, by proceeding from the finite by a limiting process, somewhat as we proceed from the notion of a sum of a finite series to the sum of an infinite series, can be so expanded that it can take care of all the topology of compact metric spaces.* Whether it is the easier method in a given

* See P. Alexandroff, Simpliziale Approximationen in der allgemeinen Topologie, Mathematische Annalen, vol. 96 (1926-27), pp. 489-511. Also see Gestalt und Lage, referred to below. 
problem is beside the point here; we can completely characterize, topologically, a compact metric space by combinatorial methods. This procedure, the spirit of which is to be found in Schoenflies' work, but whose modern form finds its inception in the work of Brouwer* and in general form in the work of Vietoris, $\uparrow$ Alexandroff, $\ddagger$ and others, will be explained later.

On the other hand, the very notion of connected itself, which as we have seen is basic in the set-theoretic topology, is a property in the large. So also is the property of nth degree connectedness, introduced by Menger. $\S$ Furthermore, in the proceedings of the 1929 congress of Slavic mathematicians, Knaster has introduced certain properties based on the notion of connectedness, which are clearly properties in the large, and which Knaster himself suggests may lead to a theory which can accomplish all that the combinatorial method has accomplished.

Despite these facts, I think it fair to say that to date the chief power of the set-theoretic method has been manifest in the investigation of local properties, whereas that of the combinatorial method has been apparent in the investigation of properties in the large. Thus, in the topology of the euclidean plane the set-theoretic method seems to have been able to attack almost any kind of problem, since here the properties in the large were restricted by the very nature of the space; in the plane a closed curve cannot be knotted, nor can two closed curves link one another, for instance. In higher dimensions, however, due to the greater degree of freedom of the space, the $i m$ grossen properties of a set become so complicated that the set-theoretic method has not been successful, by itself, in treating the prob-

${ }^{*}$ L. E. J. Brouwer, Beweis der Invarianz der geschlossenen Kurve, Mathematische Annalen, vol. 72 (1912), pp. 422-425.

$\dagger \mathrm{L}$. Vietoris, Über den höheren Zusammenhang kompakter Räume und eine Klasse von zusammenhangstreuen Abbildungen, Mathematische Annalen, vol. 97 (1927), pp. 454-472.

¥ See P. Alexandroff, Untersuchungen über Gestalt und Lage abgeschlossener Mengen beliebiger Dimension, Annals of Mathematics, vol. 30 (1928-29), pp. 101-187, as well as earlier papers referred to therein. The present citation will hereafter be referred to as Gestalt und Lage.

$\S$ See K. Menger, Dimensionstheorie,1928, p. 214, §4.

I B. Knaster, Einige Probleme über Punktmengen mit Fixpunkten, ComptesRendus du I Congrès des Mathématiciens des Pays Slaves, Warszawa, 1929 (Warsaw, 1930), pp. 287-290. See also K. Borsuk, Quelques théorèmes sur les ensembles unicohérents, Fundamenta Mathematicae, vol. 17 (1931), pp.171-209. 
lems which arise; and until the set-theoretic method has so expanded itself as to take care of these properties, it seems logical to call upon the combinatorial method for assistance.

Similarly, the combinatorial method, to date, is restricted in its application to closed sets of points, or to compact spaces, and there is no evidence, as yet, that it can be expanded so as to take care of the topology of non-compact sets, excepting, of course, certain particular cases such as the complements of closed sets in $E_{n}$ (a special case of infinite complexes).*

Is it possible for either method to become supreme in analysis situs? This question, which I think is suggested by the remarks in the preceding paragraphs, can be answered only by prophecy, and I shall not presume to prophesy. I think it fair to say, however, that if topologists will continue to restrict themselves to one method, the case for the set-theoretic method looks the more hopeful, although the difficulties encountered may be too great to make the use of the method expedient. Perhaps the settheoretic method is as yet only in its infancy; its application chiefly to the study of local properties, due to its foundation in the neighborhood idea, may be only a sign of youth. That this is not necessarily final, however, is indicated by the fact that every separable metric space can be imbedded in a compact metric space of the same dimension, and as already stated a compact metric space is amenable to combinatorial treatment.

However, until the set-theoretic method has offered a substitute, say, for the linking theorems of the combinatorial topology, and until the combinatorial method has offered a suitable method for dealing with properties of non-compact sets, I believe we must have a unified analysis situs. It is where the two methods seem to meet common ground, where one appeals to the other for assistance, that we have to deal with a unified analysis situs.

6. Evolution of the Two Methods. As is the case with many other branches of mathematics, topology had its beginnings as a means to certain ends, and it is in the beginnings of topology that we have to look for the introduction of the set-theoretic and combinatorial methods. As I have already intimated, it is in the works of Riemann and Poincaré that we find the inception of

* See Lefschetz, Topology. 
the combinatorial method. Riemann found that for the investigation of functions which arise from the integration of total differentials, it was necessary to differentiate between the various connectivities of simple surfaces; in Weber's edition of Riemann's Werke will be found a paper dealing with the notions of simply connected and $n$-fold connected surfaces, and in a Fragment will be found the essential notions embodied in the numbers of Betti (Betti's work in this connection was done without the knowledge of this Fragment, which later appeared in Weber's edition of Riemann's works). Poincaré had already employed topology in the study of differential equations when he wrote his classical memoirs on analysis situs. In these memoirs he evidently had in view principally the classification of algebraic surfaces; indeed the last two deal with the applications of analysis situs to algebraic geometry, an application that is still attracting much interest.* The combinatorial method, with its theory of connectivity and applicability in general to properties in the large, is the natural outcome of the type of problem to which Riemann and Poincaré adapted it.

As for the set-theoretic method: The neighborhood and limit point notion at the basis of the method of course formed the foundation of the point set theory as developed by Cantor and his followers, whose work looked mainly to the foundations of modern analysis. Cantor developed many theorems, which are fundamental in the present-day set-theoretic topology. An important landmark in the evolution of the set-theoretic topology, one which seems to have given it a definite direction, is the work of Schoenflies from which I have already quoted. The influence of this work on the set-theoretic work in this country and Poland is very marked, as for instance in the case of Jordan continua, the first topological investigation of which is to be found in the work of Schoenflies. It seems to have been Schoenflies' object, as indicated in the introduction of his book, to show that purely geometric problems can be treated by purely geometric methods, and he attacked the Jordan continuum, for instance, as a geometric rather than as an analytic problem. That he was correct in his viewpoint is amply attested to by the immense literature that has accumulated on the theory of Jordan con-

* See S. Lefschetz, L'A nalysis Situs et la Géométrie Algébrique, Paris, 1924. 
tinua, giving such detailed analysis of their structural properties as would hardly have been possible by the analytic method.

Although Schoenflies' investigations were devoted to the plane (so far as his principal topological results are concerned), it is noteworthy that he did not, as do the modern set-theorists to whom he gave stimulus, employ a purely set-theoretic method. Modern set-theorists have shown rather conclusively that the set-theoretic method is sufficient for most of the topology of the plane. However, Schoenflies made considerable use of the properties of polygons and of the Riemann connectivity numbers of domains bounded by polygonal configurations. Thus, the properties of domain boundaries were investigated by considering them as limiting properties of the properties of approximating polygons. Furthermore, he indicated, as quoted above, that to proceed with the study of point sets in three dimensions, it would be necessary to have a theory of the connectivity of higher dimensional polyhedra. Accordingly I feel that it is in Schoenflies' work that we find the beginnings of a unified analysis situs.

However, it is apparent that the actual motivating factor which influenced the adoption of a unified method in topology was the work of Brouwer. Brouwer was clearly influenced in his selection of problems by the work of Schoenflies, whose work he both corrected and extended. In a paper published in 1912,* he showed, using a method which has recently been generalized to higher dimensions by Vietoris, that the number of domains complementary to a continuum in the plane is a topological invariant, and that the property of being a closed curve, as defined by Schoenflies, is a topological invariant. This paper, written to supply fundamental omissions in Schoenflies' work, contains the germ of the modern adaptation of combinatorial methods to general closed sets of points, which until recently were considered open to attack only by the set-theoretic method.

In general, however, we have had two "schools" in analysis situs, the allocation of a topologist to a "school" being determined by the method that he employed. Using the set-theoretic method alone the topologist has been able to clear up the topology of the plane both as to internal and external properties, and to prove a host of internal properties of point sets in general

* Loc. cit. 
spaces-although even here, when properties in the large were concerned, the set-theoretic method has not yielded results. Using the combinatorial method alone, the topologist has been able to set up a series of invariants, especially numerical invariants such as the Betti numbers, for $n$-dimensional complexes, but has succeeded in classifying manifolds only through the two-dimensional case. The situation in combinatorial topology is indicated by two reports before the Deutsche Mathematiker-Vereinigung to which I refer below.* For the situation in set-theoretic topology I can refer the reader to the articles by Moore and Kline to which I have referred above. $\dagger$

Regarding the theory of sets of points, to which I propose to devote the remainder of this report, the set-theorist has not, in general, been able to establish external properties and properties in the large in higher dimensions. It is to this unified analysis situs that we now turn. As we proceed, it may occur to the discerning reader how ironic it is, that in the works of Poincaré and Brouwer we are today finding the tools for our attack upon that branch of mathematics, the theory of sets of points, about which Poincaré stated "later generations will regard the theory of sets of points as a disease from which one has recovered," and with which, in the sense in which we conceive of it as a formalistic theory, Brouwer will to-day have nothing to do!

\section{II}

7. General Methods. Two general kinds of unified analysis situs seem to have been employed to advantage. Suppose $S$ is a space and $M$ is a point set in $S$. In the case where $S$ is a euclidean space, I have found that to use the set-theoretic method in $M$, and the combinatorial method in $S-M$, has been quite successful in attacking such problems as the converse of the Jordan Curve Theorem in $E_{3}$, and investigating external properties of Jordan continua in $E_{n}$. However, the method which Vietoris has introduced employs the combinatorial method in $M$ by setting up certain cycles as in the pure combinatorial

* G. Feigl, loc. cit., and B. L. van der Waerden, Kombinatorische Topologie, Jahresbericht der Deutschen Mathematiker-Vereinigung, vol. 39 (1930), pp. 121-139.

$\dagger$ See also H. Tietze and L. Vietoris, Beziehungen zwischen den verschiedenen Zweigen der Topologie, Encyklopädie der Mathematischen Wissenschaften, vol. III $_{1}$, Heft 10 (1931). 
topology together with certain relations between these cycles, and, for instance, their topological limits in $M$.

It has occurred to me that I might arrange this report according to results obtained by these two methods. However, as I wish to give a general survey of the situation regarding point set theory in higher dimensions, it will perhaps be more satisfactory to proceed according to subject matter.

8. Homeomorphism Problems; Topological Definitions of $E_{n}$. Under homeomorphism problems we group those problems that concern the finding of topological properties that are necessary and sufficient in order that there exist a homeomorphism between two sets of points. For instance, if each of two sets of points is (1) a compact space and (2) irreducibly connected between two of its points, then they are homeomorphic; indeed each is homeomorphic with the set of points on the linear interval $0 \leqq x \leqq 1$, and is therefore what we call an arc. The sum of two arcs with end points in common, and only those points in common, is an $H_{1}$. Thus, of basic importance among the homeomorphism problems is the characterization of the euclidean spaces, or, preferably, of the spaces $H_{n}$ (see $\$ 4$ ). Any set of points which is homeomorphic with an $H_{n}$ is, in the analysis situs sense, itself an $H_{n}$.

Of course the topological properties which we have to impose upon a set (consisting of at least two points) in order that it be an $H_{n}$ are partially dependent upon whether we are considering the set as a general topological space in itself, or as a subset of another space whose properties we are given. Suppose, for instance, that we are dealing with subsets of locally compact spaces; we can state the two following sets of properties as characterizing the $H_{n}$ indicated:

$H_{1}$ : Connected, locally connected, not disconnected by the omission of any $\left\{\begin{array}{l}\text { point } \\ 0 \text {-cell }\end{array}\right\}$, but disconnected by the omission of any $\left\{\begin{array}{c}\text { two points } \\ 0 \text {-sphere }\end{array}\right\} *^{*}$

$\mathrm{H}_{2}$ : Compact, connected and locally connected, not disconnected by the omission of any $\left\{\begin{array}{c}\text { arc } \\ 1 \text {-cell }\end{array}\right\}$, but disconnected by the

* See R. L. Wilder, Concerning simple continuous curves and related point sets, American Journal of Mathematics, vol. 53 (1931), pp. 39-55. 
omission of any $\left\{\begin{array}{c}\text { simple closed curve } \\ 1 \text {-sphere }\end{array}\right\} *^{*}$

Both of these characterizations have been obtained by the set-theoretic method, and are thus ordinarily stated by reading the upper line in the braces. I have, however, indicated in the lower lines of the braces the corresponding combinatorial terms; perhaps we should, instead of " 0 -sphere" and "1-sphere," say "0-manifold" and "1-manifold." The following problems suggest themselves: Writing " $H_{0}$ " for " 0 -sphere," etc., do we get an inductive definition of $H_{n}$ ? Assuming the definition of $H_{i}, i<n$, the $(n-1)$-cell is one of the parts into which an $H_{n-2}$ divides $H_{n-1}$; thus, is a Jordan continuum which is not disconnected by the omission of any $(n-1)$-cell, but is disconnected by the omission of any $H_{n-1}$, an $H_{n}$ ? Obviously not in the general case, at least not without the existence in the set of an $H_{n-1}$. In particular, then, what conditions in addition to being a Jordan continuum which contains an $\mathrm{H}_{2}$, not disconnected by the omission of any 2-cell but disconnected by the omission of any $\mathrm{H}_{2}$, are necessary and sufficient in order that we have an $H_{3}$ ?

The most elementary definition of $H_{n}$ was indicated by Tietze, $\dagger$ and is based upon the consideration of $H_{n}$ as the product space of the already topologically defined $H_{1}$. A distinct disadvantage, perhaps, of this definition, is that the neighborhoods must be defined in terms of sets of $n$ neighborhoods of $H_{1}$.

The definition of $H_{n}$ given by Alexandroff $\ddagger$ starts with a general topological space $S$, and depends upon the existence, in $S$, of a sequence (spectrum) of finite sets of points, $F, F_{1}$, $F_{2}, \cdots, F_{k}, \cdots$, called nets. The initial set $F$ consists of $n+2$ points, and every subset of $F$ consisting of $n+1$ points is called a scaffold; thus the points of $F$ correspond to the $n+2$ vertices

* See L. Zippin, On continuous curves and the Jordan curve theorem, American Journal of Mathematics, vol. 52 (1930), pp. 331-350.

$\dagger$ H. Tietze, Über Analysis Situs, Abhandlungen aus dem Mathematischen Seminar der Hamburgischen Universität, vol. 2 (1923), pp. 37-68. If $X$ and $Y$ are spaces, the product space $X \cdot Y$ is a space whose points are the pairs $(x, y)$ of points of $X$ and $Y$, and for which a neighborhood of a point $\left(x_{0}, y_{0}\right)$ is the set of points $(x, y)$ such that $x$ and $y$ belong respectively to neighborhoods of $x_{0}$ and $y_{0}$. See also K. Yoneyama, The conception of a curve, a surface and a solid, Memoirs of the Kyoto College of Science and Engineering, vol. 5 (1912-13), pp. 261-269, for an inductive building up of the euclidean element.

$\ddagger$ P. Alexandroff, Zur Begr ündung der n-dimensionalen mengen-theoretischen Topologie, Mathematische Annalen, vol. 94 (1925), pp. 296-308. 
that we get in the division of $H_{n}$ into $n+2 n$-cells. The set $F_{1}$ consists of $(n+2) \cdot(n+1)$ ! scaffolds such as we get upon the barycentric subdivision* of the above cellular subdivision of $H_{n}$. In general, each $F_{k}$ consists of scaffolds of $n+1$ points each, and the spectrum is so related to the space $S$ by conditions on the closures of any scaffold of $F_{k}$ and its derived (analogous to barycentric subdivision) scaffolds, that the space $S$ is homeomorphic with the $n$-dimensional space $H_{n}$. It will be noticed that although this definition of $H_{n}$ is entirely set-theoretic in form, its spirit is combinatorial in the sense that it preserves the analogy to the cellular subdivision of $H_{n}$ and its continued barycentric subdivision. In short, it translates a fundamental combinatorial method into set-theoretic form, and is in this sense an achievement of a unified analysis situs.

Unfortunately limitations of space do not permit my going into detail concerning various definitions of $H_{2}$ other than that of $\mathrm{Zippin}$ given above. $\dagger$ And as to the progress that has been made on the homeomorphism problem relative to the $n$-dimensional manifold, I may refer my readers to the report by van der Waerden already cited above.

9. External Properties of the Arc, Simple Closed Curve and Simple Closed Surface in $E_{n}(n>1)$. I have indicated above, in quoting from Moore's Kansas lecture, that the fundamental problems here have been largely cleared up. In the case of the simple closed surface (that is, $H_{2}$ ), Brouwer $\ddagger$ showed in 1912 that an $(n-1)$-dimensional manifold imbedded in $E_{n}$ determines two domains, of which it is the common boundary, and in an accompanying paper§ established the accessibility of the manifold. Furthermore, since the sets mentioned in the heading

* Or regular subdivision; see Veblen, loc. cit.

† See R. L. Moore and J. R. Kline, this Bulletin, vol. 28 (1922), p. 380, abstract No. 7; T. Radó, Über den Begriff der Riemannsche Fläche, Acta Litterarum ac Scientiarum, Szeged, vol. 2 (1925), pp. 101-121; I. Gawehn, Über unberandete 2-dimensionale Mannigfaltigkeiten, Mathematische Annalen, vol. 98 (1928), pp. 321-354; C. Kuratowski, Une caractérisation topologique de la surface de la sphère, Fundamenta Mathematicae, vol. 13 (1929), pp. 307-318; J. H. Roberts, $A$ point set characterization of closed 2-dimensional manifolds, ibid., vol. 18 (1932), pp. 39-46.

$\ddagger$ L. E. J. Brouwer, Beweis des Jordanschen Satzes für den n-dimensionalen Raum, Mathematischen Annalen, vol. 71 (1912), pp. 314-320.

$\S$ L. E. J. Brouwer, Über Jordansche Mannigfaltigkeiten, ibid., pp. 320-327. 
of this section are, respectively, the 1-cell, $H_{1}$, and $H_{2}$, and accordingly complexes in the combinatorial sense, the duality theorem of Alexander explained above (\$4) embodies fundamental results concerning their external properties. As the Betti numbers of the 1-cell are all zero, the arc neither separates $E_{n}$ nor is it linked by any cycle in its complement. The Betti numbers of $H_{1}$ being all zero, except $p^{1}$, which is 1 , the simple closed curve is linked only by one cycle, an $(n-i-1)$-cycle, of its complement, that is, it separates $E_{2}$ into just two domains (see the Jordan Curve Theorem); in $E_{3}$ it does not separate space but is linked by a 1-cycle, etc. In the case of $H_{2}$ (simple closed surface), the Betti numbers are all zero except $p^{2}$ which is equal to 1 , and the reader can easily supply his own conclusions as to space separation and linking.

The accessibility of the arc, as well as the accessibility of the $i$-cell $(i<n)$ imbedded in $E_{n}$, is a special case of a theorem of Mazurkiewicz* to the effect that any closed subset of $E_{n}$ which is homeomorphic with a subset of $E_{n-1}$ is accessible from its complement. Indeed, I have been able to show, $\nmid$ by strong use of the combinatorial properties of the complement of the set, that such a set is regularly accessible from its complement.

The theorem that the domains determined by a simple closed curve in $E_{2}$ are uniformly locally connected, established by Moore, I have extended to $E_{n}$ by determining that the domains bounded by an (n-1)-manifold are uniformly locally connected. $\ddagger$ This theorem is also capable of extension in another direction (see $\$ 10$ ).

It will be admitted, I think, that our knowledge of the external properties of the arc, simple closed curve, and surface in $E_{n}$ is today quite extensive. Thus, in $E_{3}$, the simple closed surface is the common boundary of just two domains, from each of which it is accessible, and each of which is uniformly locally connected, and is not linked by any 1-cycle of its complement (for the converse of this theorem see $\$ 10$ ), properties which have been found by the combined use of set-theoretic and combina-

* S. Mazurkiewicz, Sur un problème de M. Knaster, Fundamenta Mathematicae, vol. 13 (1929), pp. 146-150.

$\dagger$ R. L. Wilder, Extension of a theorem of Mazurkiewicz, this Bulletin, vol. 37 (1931), pp. 287-293.

$\ddagger \mathrm{R}$. L. Wilder, A converse of the Jordan-Brouwer separation theorem in three dimensions, Transactions of this Society, vol. 32 (1930), pp. 632-657, §1. 
torial methods. To be sure, the theorem of Schoenflies regarding the extension of the homeomorphism between two simple closed curves of $E_{2}$ to the entire plane does not go over to $H_{2}$ 's in $E_{3},{ }^{*}$ but certainly we are not warranted in saying that the point set theorems of the plane do not extend to higher dimensions.

10. The Jordan Continuum; Extension of Schoenflies' Results to Higher Dimensions. For some time it has been an open question with the set-theorist as to just how much may be done with the theory of Jordan continua in $E_{n}(n>2)$, particularly in the matter of external properties. If the set-theoretic method alone is used, the prospects do not seem hopeful, but from the standpoint of unified analysis situs I believe a great deal can be done in extending the theory that Schoenflies built up in the plane.

One of Schoenflies' best known results is the converse of the Jordan Curve Theorem. Recognizing that not all closed curves are simple closed curves, Schoenflies set himself the problem of so extending the Jordan Curve Theorem as to get conditions that are sufficient, as well as necessary, in order that a point set be a simple closed curve. He found that, in addition to the simple closed curve being the common boundary of two domains (Jordan Curve Theorem), it is also accessible from each of its complementary domains, and this condition proved sufficient for a converse theorem. The set-theorist has taken the attitude that in getting a converse in three dimensions for the simple closed surface $\left(\mathrm{H}_{2}\right)$, one should look for a stronger kind of accessibility. Up to date such a procedure has not been found successful, $\dagger$ and I do not feel as I did formerly that such a procedure is really following out the spirit of Schoenflies' ideas. In view of the fact that he himself suggests the necessity of having regard for the higher connectivity of three-dimensional space (see quotation in \$3), in extending his results to higher dimensions, it certainly seems to be appropriate to incorporate such information as is contained in the Alexander Duality Theorem in a converse of the theorem concerning the separation of $E_{3}$ by

* As shown by J. W. Alexander, An example of a simply connected surface bounding a region which is not simply connected, Proceedings of the National Academy of Sciences, vol. 10 (1924), pp. 8-10.

$\dagger$ A special case of the converse, employing a conical accessibility, has been announced by T. C. Benton, this Bulletin, vol. 37 (1931), p. 34, abstract No. 80. 
a simple closed surface (see last paragraph of $\S 9$ ). By following such a procedure, I have been able to get not only such a converse theorem, but have also found* that it is a special case of the converse of the theorem concerning the separation of $E_{3}$ by a closed two-dimensional manifold of genus $k$. This converse may be stated as follows. In $E_{3}$, let $K$ be a closed and bounded point set such that (1) the Betti number $p^{0}\left(E_{3}-K\right) \geqq 1$, and the Betti number $p^{1}\left(E_{3}-K\right)$ is finite; and (2) if $D$ is a domain complementary to $K$, then $D$ is uniformly locally connected and every point of $K$ is a limit point of $D$. Then $K$ is a closed twodimensional manifold of genus $k=\frac{1}{2} p^{1}\left(E_{3}-K\right)$. By setting $p^{1}\left(E_{3}-K\right)=0$ in this theorem we of course get the converse of the theorem regarding the separation of $E_{3}$ by a simple closed surface. $\dagger$

No converse theorem for $H_{n-1}$ in $E_{n}$, where $n>3$, has as yet been found. Apparently the converse announced by Rey Pastor recently $\ddagger$ cannot be true, even in $E_{3}$, in view of the example of Alexander cited above.

Another fundamental result of Schoenflies is that a continuum $K$ in $E_{2}$, the diameters of whose complementary domains form a zero sequence and the boundaries of whose complementary domains are accessible from all sides, is a Jordan continuum. R. L. Moore has shown by examples§ that this theorem is not

* See this Bulletin, vol. 36 (1930), p. 219, abstract No. 196; for even weaker conditions, see ibid., vol. 37 , p. 519 , abstract No. 236.

$\dagger$ See also my paper $A$ converse of the Jordan-Brouwer $\cdots$, loc. cit. I have recently come across an early attempt at the converse, for the case of an $H_{2}$ in $E_{3}$, in a paper by $\mathrm{K}$. Kaluzsay, $A$ feluiletre vonatkozó Jordan-têtel megforditása, Mathematikai és Physikai Lapok, vol. 24 (1915), pp. 101-141. Upon obtaining a translation of Kaluzsay's results, I have found the interesting fact that his conditions (No. 5 apparently gives the uniform connectedness im kleinen of the complementary domains) closely approximate those which I gave in the Transactions paper just cited, except that instead of the condition on the Betti number which I used, he assumed that (condition 3 ) any closed polygon in a complementary domain can be continuously deformed into a point in that domain, thus yielding only a special case.

$\ddagger \mathrm{J}$. Rey Pastor, Une propriété caractéristique des variétés de Jordan, Comptes Rendus, vol. 192 (1931), pp. 27-29. For further commentary on this paper, see L. E. J. Brouwer, Über freie Umschliessungen im Räume, Proceedings of the Royal Academy of Amsterdam, vol. 34 (1931), pp. 100-101.

$\S$ On the relation of a continuous curve to its complementary domains in space of three dimensions, Proceedings of the National Academy of Sciences, vol. 8 (1922), pp. 33-38. 
true as it stands in $E_{3}$, and a characterization of Jordan continua by their external properties in $E_{3}$ is lacking. The chief reason for this is undoubtedly that not enough of the external properties have as yet been found, and some of these at least will be of a combinatorial nature. The most natural approach to the problem would probably be to consider first those Jordan continua that are the common boundaries of just two complementary domains. It is known that in such a case the possession of the property of being uniformly locally connected by the two domains is sufficient to make the common boundary a Jordan continuum,* but this is not a necessary condition.

To illustrate the complexities of the problem: In $E_{2}$ a Jordan continuum which is the common boundary of two domains is a simple closed curve $\left(H_{1}\right)$. I have found $\dagger$ an example, however, in $E_{3}$, of a Jordan continuum which is the common boundary of three (any finite number, or a denumerable infinity of) domains, all of which are uniformly locally connected. On the other hand, I have found that if $K$ is the common boundary of two uniformly locally connected domains $D_{1}$ and $D_{2}$ in $E_{3}$, and there exists a point $P$ of $K$ and a positive number $\epsilon$ such that all 1-cycles of $D_{i} \cdot S(P, \epsilon),(i=1,2)$, are homologous to zero in $D_{i}$, then $K$ is the common boundary of only two domains. Thus, to be a common boundary of at least three such domains, a Jordan continuum must be linked by 1-cycles in every neighborhood of every point of the continuum. It would seem that if we do not allow a Jordan continuum which is a common boundary of two domains to be linked at all by cycles of dimension greater than zero, it is probably very greatly restricted. In particular, I have found that if a Jordan continuum which is the boundary of a domain $D$ in $E_{n}$ has no cut point (such is always the case when it is the common boundary of two domains), then, if the $(n-2)$ cycles of $D$ all bound in $D$, they bound uniformly in $D$.

Before leaving this matter of the external properties of Jordan continua in $E_{n}$, I want to emphasize two matters. First, that the connectedness of a domain is a special case of the bounding properties of its $i$-cycles; in this sense the result stated at the end of the preceding paragraph is the extension to $E_{n}$ of the uni-

* See R. L. Moore, On the relation . . . loc. cit., Theorem 1, and my paper A converse of the Jordan-Brouwer . . ., loc. cit., p. 644, II.

$\dagger$ See this Bulletin, vol. 37 (1931), p. 525, abstract No. 258. 
formly locally connected property of the domains bounded by a simple closed curve in $E_{2}$ (see third paragraph of $\$ 9$ ). Second, separation of $E_{n}$ by a closed set of points $K$ is equivalent to the linking of 0-cycles of the complement, and hence a special case of the linking of the set by $i$-cycles of its complement. By keeping these two matters in mind, many plane theorems can be extended to higher dimensions. To illustrate the second, consider the MooreKuratowski theorem to the effect that if a Jordan continuum separates two points $P$ and $Q$ in $E_{2}$, then it contains a simple closed curve which separates them. That this theorem does not generalize to three dimensions as a separation theorem is well known. However, as a linking theorem it generalizes* to any number of dimensions as follows. If, in $E_{n}$, an $(n-2)$-cycle links a Jordan continuum $M$, then it links a simple closed curve in $M$. Furthermore, in connection with a result of Mooret to the effect that in $E_{2}$ the set of all points on all simple closed curves of a Jordan continuum $M$ that enclose a given point $P$ is the sum of a finite number of Jordan continua, we have, in $E_{n}$, the result that the set of all points that lie on simple closed curves of a Jordan continuum $M$ that are linked by an $(n-2)$-cycle is the sum of a finite number of Jordan continua each of which consists of a finite set of true cyclic elements of $M$.

So far we have considered only external properties of Jordan continua, and I hope I have made it clear that by taking a combinatorial point of view in the complement of the continuum (all of the above results were obtained by using the set-theoretic method in the continuum), the prospect of extending theorems for the plane to higher dimensions and of determining external properties of Jordan continua, is very hopeful.

Let us now consider the internal structure of Jordan continua. The modern Polish and American set-theorists have used the set-theoretic method exclusively in investigating internal properties, the majority of their results extending to general spaces since the continuum is usually considered as a space in itself without reference to any imbedding space. The culmination of

* See my paper On the linking of Jordan continua in $E_{n}$ by $(n-2)$-cycles, to appear soon in the Annals of Mathematics.

$\dagger \mathrm{R}$. L. Moore, Concerning paths that do not separate a given continuous curve, Proceedings of the National Academy of Sciences, vol. 12 (1926), pp. $745-753$, Theorems 7 and 8 . 
the attempt to divide the Jordan continuum in to elements is the cyclic element theory of Whyburn,* which furnishes what we might call the atomic structure of the Jordan continuum in terms of which it takes on the semblance of an acyclic Jordan continuum. However, in higher dimensions the cyclic element, which is itself a Jordan continuum, may have a highly complicated structure, and the question arises as to whether there may not be devised some sort of electronic structure for it. Since the basis for the true cyclic element is the simple closed curve, or 1-cycle, I would suggest that we ought also to investigate the structure of the Jordan continuum with reference to its cycles of higher dimension. Of course I am not referring here to the geometric cycle of the combinatorial topology; rather am I referring to the complete cycles introduced by Vietoris, $\uparrow$ which are based on the pure combinatorial notion of cycle. First, however, let me give a short exposition of this theory.

Let $M$ be any closed subset of $E_{n}$; a set of $k+1$ points of $M$ is called a $\delta$-simplex of $M$ of dimension $k$ if the diameter of the set is $<\delta$. (Singularities may occur when points fall together.) The notion of a complex made up of such simplexes, called a $\delta$ complex, of the boundary of such a complex, of $\delta$-cycles, bounding relations, etc., are set up as usual. A $\delta$-cycle of $M$ is called $\epsilon$-homologous to zero in $M$ if it is the boundary of an $\epsilon$-complex in $M$. We then may set up the notion of $\epsilon$-independence of $\delta$-cycles with respect to homologies, and the corresponding Betti numbers.

If $\epsilon_{i},(i=1,2,3, \cdots)$, is a sequence of positive numbers such that $\lim \epsilon_{i}=0$, and $r=\left(r_{1}, r_{2}, r_{3}, \cdots\right)$ is a sequence of $r$ dimensional $\epsilon_{i}$-cycles, $r_{i}$, in $M$, then $r$ is called an $r$-dimensional complete cycle of $M$ if for every positive number $\epsilon$ there exists a number $N$ such that for $k>N, \epsilon_{k}<\epsilon$, while for every two numbers $k_{1}$ and $k_{2}$ which are greater than $N$,

$$
\stackrel{\epsilon}{r_{k_{1}} \sim r_{k_{2}}}
$$
in $M$.

Two complete cycles $r$ and $r^{\prime}$ are called homologous if for every positive number $\epsilon$ there exists an $N$ such that for all $k>N$,

* See C. Kuratowski and G. T. Whyburn, Sur les éléments cycliques et leurs applications, Fundamenta Mathematicae, vol. 16 (1930), pp. 305-331.

† See Vietoris, loc. cit., and Alexandroff, Gestalt und Lage, loc. cit. 


$$
r_{k} \stackrel{\epsilon}{\sim} r_{k}^{\prime},
$$

in $M$.

Now, as to the relation between the closed subsets of $M$, and these complete cycles and their homologies: If $F$ is a closed subset of $M$ and $r$ is a complete cycle of $M$, then, if $r$ is also a complete cycle of $F, F$ is called a carrier* of $r$. The common part of all carriers of $r$ is also a carrier of $r$ and is called the smallest carrier of $r$. We introduce also the notion of carrier of a homology. A complete cycle $r$ is homologous to zero in a closed set $F$ if each $r_{i}$ bounds an $\eta_{i}$-complex $K_{i}$ in $F$ such that $\lim \eta_{i}=0$. Every subset $F^{\prime}$ of $F$ which is a topological limit of a convergent subsequence $\dagger$ of such sets $K_{i}$ is called a carrier of the homology $r \sim 0$ in $M$.

Let us call a complete cycle essential if it has at least one carrier in which it is not homologous to zero. Then, for instance, every simple closed curve of a Jordan continuum $M$ is the carrier of an essential 1-dimensional complete cycle $C^{1}$ of $M$. If $C^{1} \sim 0$ in $M$, every carrier of this homology contains an irreducible membrane $\ddagger$ with respect to $C_{1}$; that is, a closed subset of $M$ which is irreducible with respect to the property of being a carrier of this homology. This irreducible membrane cannot be disconnected by the omission of an at most 0-dimensional subset, and if it is not of higher dimension than 2 , is a 2-dimensional cantorian manifold. $\$$

We can also introduce the notion of two simple closed curves $J$ and $K$ of a Jordan continuum $M$ being equivalent, or homologous to one another, if complete cycles of which they are the respective carriers are homologous to one another in $M$.

In like manner we can consider the higher dimensional complete cycles of $M$ and their carriers. I believe that a consideration of these cycles, related cantorian manifolds, and of the linkings by cycles of the complement of $M$, might lead to a considerable theory of Jordan continua in higher dimensions.

Aside from the theory of Jordan continua, the irreducible membrane, being a generalization of the irreducible continuum,

* Träger; see Gestalt and Lage, pp. 168-169.

$\dagger$ See Hausdorff, Mengenlehre, p. 146.

¥ See Gestalt und Lage, p. 179.

$\S$ As defined by Urysohn, an $n$-dimensional cantorian manifold is an $n$ dimensional closed point set which is not disconnected by the omission of any of its at most $(n-2)$-dimensional closed subsets. 
offers great possibility of investigation of structural properties by the unified method.*

11. Continua. Concerning the internal structure of continua that are not necessarily Jordan continua we find a notable collection of papers in the set-theoretic topology. For instance a decomposition in to elements, such as the upper semi-continuous collections of continua of Moore, similar to the decomposition of the Jordan continuum into cyclic elements, has been studied by several authors. Limitations of space forbid my giving an exposition of what has been done along this line. $\dagger$ Since, in taking up properties of continua that are imbedded in $E_{n}$, I shall wish to make use of certain combinatorial properties of general closed sets, it will be convenient to go directly to the latter.

12. Closed Sets in General. The most important result which has recently been obtained I believe to be a duality theorem, proved independently by Frankl, Alexandroff, and Lefschetz.

Let $M$ be any closed subset of $H_{n}$. We then define complete cycles in $M$ as above. A system of complete cycles of $M$,

$$
r_{1}, r_{2}, r_{3}, \cdots, r_{m}, \cdots,
$$

is called a basis for the r-dimensional complete cycles of $M$ if the cycles (1) are linearly independent in the sense that no finite linear combination of them is homologous to zero in $M$, and if for every $\epsilon$ there is a finite subsystem $\left(1^{\prime}\right)$ of (1) such that every

* See Gestalt und Lage, pp. 179-181.

† I believe that Moore's recent colloquium lectures on point set theory treat of decompositions of continua rather extensively. It may be of interest to call attention here to results of W. Hurewicz, Über oberhalbstetige Zerlegungen von Punktmengen in Kontinua, Fundamenta Mathematicae, vol. 15 (1930), pp. 57-60, to the effect that an upper-semicontinuous decomposition of a closed set in $E_{2}$ constitutes an at most 2-dimensional space, whereas in $E_{3}$ there exists a curve $M$ such that for every compact space $S$ there is a subset $M_{S}$ of $M$ and an upper-semicontinuous decomposition of $M_{S}$ which is homeomorphic with $S$.

$\ddagger$ F. Frankl, Topologische Beziehungen in sich kompakter Teilmengen euklidischen Räume zu ihren Komplementen sowie Anwendung auf die Prim-EndenTheorie, Wiener Akademie der Wissenschaften, Math.-Naturw. K1., Sitzungsberichte, Abt. 2A, vol. 136 (1927), pp. 689-699; Gestalt und Lage, pp. 156 ff. (also see Göttinger Nachrichten, Math.-Phys. Kl., Nov. 25, 1927); S. Lefschetz, Closed point sets on a manifold, Annals of Mathematics, vol. 29 (1928), pp. 232-254, as well as earlier papers referred to therein. 
$r$-dimensional complete cycle is $\epsilon$-homologous to a linear combination of $\left(1^{\prime}\right) . *$ The number of cycles in (1) is a topological invariant of $M$, and may be called the $r$-dimensional Brouwer number (since for the case $n=2$ and $r=1$ it was originally set up by Brouwer) or the $r$-dimensional Betti number of $M$. I shall use the latter terminology. $\dagger$

A system of $(n-r-1)$-dimensional cycles (geometric) of $H_{n}-M$

$$
\Gamma_{1}^{n-r-1}, \Gamma_{2}^{n-r-1}, \cdots, \Gamma_{m}^{n-r-1}, \cdots,
$$

is called an $(n-r-1)$-dimensional basis for $H_{n}-M$ if no finite linear combination of them is homologous to zero in $H_{n}-M$ and every $(n-r-1)$-dimensional cycle of $H_{n}-M$ is homologous to a finite linear combination of them.

Let $r$ be an $r$-dimensional complete cycle in $M$, and $\Gamma^{n-r-1}$ an $(n-r-1)$-cycle of $H_{n}-M$. Then $r$ and $\Gamma^{n-r-1}$ will be said to be linked if $\Gamma^{n-r-1}$ links almost all of the $\epsilon_{i}$-cycles that make up $r$, when these are geometrically realized. Then the bases (1) and (2) may be so chosen that their cycles are uniquely linked; that is, $r_{m}$ and $\Gamma_{m}{ }^{n-r-1}$ are linked, but $r_{m}$ and $\Gamma_{i}{ }^{n-r-1}$ for $i \neq m$ are not linked. Consequently, if, in the Alexander Duality Theorem, $M$ stands for a closed set of points, the theorem still holds. And as the Betti number of $M$ is an invariant, the number $p^{n-r-1}$ $\left(H_{n}-M\right)$ is an invariant. One of the most important consequences of this is obtained for the case $r=n-1$, in which we obtain the result that the number of domains complementary to a closed set of points is invariant. Here we have again an extension to $n$ dimensions of an important theorem of plane topology, this theorem having been proved for the number of domains complementary to a closed set in $E_{2}$ by Brouwer in 1912. $\ddagger$

In case a closed set $M$ separates two points $P$ and $Q$ of $E_{n}$,

* See Gestalt und Lage, pp. 159-160, and Vietoris, loc. cit., part II.

† Obviously $p^{0}(M)+1$ is the number of components of $M$. It may be of interest to point out here a relation between this part of the theory of complete cycles and the point set theory of Cantor, namely, that the Cantor definition of connectedness, defined in terms of " $\epsilon$-chains," demanded, if translated into combinatorial terminology, that the 0-dimensional complete cycles of $M$ should all be homologous to zero in $M$.

$\ddagger$ Beweis der Invarianz der geschlossenen Kurve, loc. cit. 
then, as has been shown by Mazurkiewicz, ${ }^{*} M$ contains a set $F$ which is an irreducible cut between $P$ and $Q$. Since a common boundary of two domains in $E_{n}$ is necessarily an irreducible cut between points in these respective domains, it follows from Mazurkiewicz' result that a necessary and sufficient condition that a set $M$ in $E_{n}$ be an irreducible cut between some two points is that it be the common boundary of two domains. That an irreducible cut between two points is a continuum follows from the Phragmen-Brouwer Theorem, about which we shall speak below when we consider separation theorems.

As separation of $E_{n}$ is equivalent to linking by 0 -cycles, the result just stated may be formulated as follows. In order that a set $M$ in $E_{n}$ should be irreducibly linked with a 0 -cycle of $E_{n}-M$ it is necessary and sufficient that $M$ be the common boundary of two domains. The question arises, is the property of a set $M$ being the common boundary of two domains invariant with the set $M$ ? And can a necessary and sufficient condition for a set $M$ being irreducibly linked with an $r$-cycle of $E_{n}-M$, where $r$ is not necessarily equal to 0 , be obtained? By making use of the theory of the complete cycles of a closed set, Alexandroff has obtained $\dagger$ the answers to these questions, by getting the necessary and sufficient condition asked for, and from this deducing that the existence of an $r$-cycle irreducibly linked with $M$ is topologically invariant with $M$; as a particular case of this, the property of being a common boundary of two domains of $E_{n}$ is a topological invariant of $M$.

However, a set $M$ can be an irreducible cut between two points without being a completely irreducible cut $\ddagger$ of $E_{n}$. For the case of $E_{2}$, Kuratowski has shown $\S$ that if a set $M$ which cuts the plane has only a finite number of complementary domains,

* Fundamenta Mathematicae, vol. 1 (1920), p. 63. A set $F$ is an irreducible cut of $E_{n}$ between $P$ and $Q$ if it separates $P$ and $Q$ but no proper closed subset of $F$ separates $P$ and $Q$. The generalization of this notion to that of a set $F$ which is irreducibly linked by a cycle of $E_{n}-F$ is obvious, separation being a special case of linking (see $\$ 10$ ).

$\dagger$ Gestalt und Lage, pp. $169 \mathrm{ff}$.

$\ddagger$ A cut $F$ is a completely irreducible cut of $E_{n}$ if no proper closed subset of it separates points of $E_{n}$. Thus, a simple closed curve in $E_{2}$ is a completely irreducible cut of $E_{2}$.

$\S \mathrm{C}$. Kuratowski, Sur les coupures irréductibles du plan, Fundamenta Mathematicae, vol. 6 (1924), pp. 130-145. 
then it contains a completely irreducible cut of the plane, and points out that if the number of domains is infinite, there may be no such cut. However, if $M$ is a Jordan continuum that cuts the plane, it does contain a completely irreducible cut, namely an $H_{1}$. In the case of $E_{n}$, it is still true (following Kuratowski's proof, which depends only on Mazurkiewicz' theorem) that if $M$ has only a finite number of complementary domains, it contains a completely irreducible cut of $E_{n}$. In view of what I have said in $\$ 10$ concerning separation of $E_{n}$ being a special case of linking, problems arise as to the extension of these theorems to theorems concerning the linking of a set by $r$-cycles, $r>0$.

Let us consider, first, the notion of a closed m-dimensional cantorian manifold, introduced by Alexandroff.* A closed set of points of dimension $m$ whose $m$ th Betti number is not zero, while the $m$ th Betti number of each of its proper closed subsets is zero, is called a closed $m$-dimensional cantorian manifold. By virtue of the invariance of the Betti numbers, the property of being a closed $m$-dimensional cantorian manifold is a topological invariant. And in view of the duality theorem mentioned above, the completely irreducible cuts of $E_{n}$ are identical with the closed $(n-1)$-dimensional cantorian manifolds of $E_{n}$, and thus are identical with those sets that separate $E_{n}$ and are the common boundaries of all their complementary domains. Thus we have a characterization of these manifolds by their external properties, something we lack for the combinatorial manifolds, excepting, of course, the case of the 1-manifold in $E_{2}$ (Schoenflies' converse of the Jordan Curve Theorem) and the 2-manifold in $E_{3}$ (see $\$ 10$ ).

To return to the problems mentioned above: It follows at once, from an induction theorem of Brouwer, $\dagger$ that if a closed set $M$ is linked by an $r$-cycle, then it contains a set $F$ which is irreducibly linked by this $r$-cycle. Regarding the generalization of the theorem of Kuratowski regarding completely irreducible cuts of $E_{2}$, I have shown that if a closed set $M$ is $r$-dimensional and is linked by only a finite number of $(n-r-1)$-cycles, then it contains a closed $r$-dimensional cantorian manifold. As a special

* Gestalt und Lage, p. 176.

$\dagger \mathrm{L}$. E. J. Brouwer, On the structure of perfect sets of points, Proceedings of the Amsterdam Academy, vol. 14 (1912), pp. 137-147. 
case of this theorem it follows that if a set separates $E_{n}$ into a finite number of domains, then it contains a completely irreducible cut of $E_{n}$ (the hypothesis as to the dimensionality of $M$ being superfluous in this case). The problem as to whether the restriction that $M$ be linked by only a finite number of $(n-r-1)$ cycles is necessary in case $M$ is a Jordan continuum remains open, except, of course, for the case $r=1$ (see $\$ 10$ ).

The case of a bounded set $C$ which is the common boundary of two (or more) domains in $E_{n}$ was considered by Urysohn in his original memoirs on dimension theory. Urysohn showed that, in $E_{3}$, the set $C$ is a 2-dimensional cantorian manifold and indicated a general plan for proof of the fact that, in $E_{n}$, the set $C$ is an $(n-1)$-dimensional cantorian manifold. A complete proof of the latter fact was later given by Alexandroff, who furthermore showed that if $K$ is a closed subset of $C$ such that $p^{n-2}(K)=0$, then $C-K$ is connected.* I have recently proved a theorem which contains both of these results as special cases, namely, that if $p^{n-2}(K)=k$, then $C-K$ has at most $k+1 \mathrm{com}$ ponents; and if $C$ is a common boundary of at least three domains, then $C-K$ has at most $k$ components. In this same connection I have also shown that if $M$ is any closed $m$-dimensional cantorian manifold and $K$ a closed subset of $M$ such that $p^{m-1}(K)=k$, then $M-K$ has at most $k+1$ components; and that if $p^{m}(M)>1$ and $p^{m-1}(K)>0$, then $M-K$ has at most $k$ components.

In regard to those closed sets that are characterized only by their being boundaries of domains, important results are embodied in the extension by Kaufmann $\dagger$ of the Carathéodory theory of prime ends to $E_{3}$. The details of this are too long to include here. Instead of four types of prime ends, as set up by Carathéodory for $E_{2}$, Kaufmann distinguishes six types in $E_{3}$.

The accessibility of general closed sets in $E_{n}$ has been studied to a certain extent. I have spoken above of the accessibility theorems obtained by Mazurkiewicz and myself. In general, it

* Gestalt und Lage, p. 154.

$\dagger$ Boris Kaufmann, Über die Berandung ebener und raumlicher Gebiete (Primendentheorie), Mathematische Annalen, vol. 103 (1930), pp. 70-144. See also $\mathrm{H}$. Teresaka, On the boundary of open surface, Japanese Journal of Mathematics, vol. 8 (1931), pp. 49-64. 
is well known, as a result of a standardized procedure for proving accessibility, that a set $M$ which does not separate any domain of $E_{n}$ is accessible at all its points. Thus, since Urysohn showed* that no $F_{\sigma}$ which is at most $(n-2)$-dimensional cuts a domain, it follows that every point of such a set is accessible from its complement. In particular, every at most $(n-2)$-dimensional closed set is accessible from its complement.

Further results on closed sets will be mentioned below in other connections.

13. Separation Theorems. $\dagger$ From a set-theoretic point of view, these were reported upon by Kline. I wish, however, to point out an important set of addition theorems, obtained by the combinatorial method, which are fundamental in the investigation of separation (and linking) properties of closed sets in higher dimensions.

The model for all later addition theorems is that of Alexander, $\ddagger$ which states that if $M$ is the sum of two closed sets $A_{1}$ and $A_{2}$ in $E_{n}$, and $\Gamma^{i}$ an $i$-cycle of $E_{n}-M$ such that there is an $(i+1)$-complex $L_{j}{ }^{i+1}$ bounded by $\Gamma^{i}$ in $E_{n}-A_{j}(j=1,2)$, and such that the cycle $L_{1}{ }^{i+1}+L_{2}{ }^{i+1}$ bounds in $E_{n}-A_{1} \cdot A_{2}$, then the cycle $\Gamma^{i} \sim 0$ in $E_{n}-M$. This is called an addition theorem since non-linking by each of two sets is extended, under the given assumptions, to non-linking by their sum. The set-theorist will recognize immediately that the classical Phragmen-Brouwer theorem, which states that if neither of two mutually exclusive bounded closed sets separates the plane, their sum does not, follows at once, and for $E_{n}$, from this theorem-a fact which probably led Alexandroff to apply it to obtain vast generalizations of the Phragmen-Brouwer Theorem for $E_{n}$.

From the theorem just stated, and from the duality theorem for closed sets, it follows that if $A_{1}$ and $A_{2}$ are closed sets in $E_{n}$, and a cycle $\Gamma^{i} \sim 0$ in both $E_{n}-A_{1}$ and $E_{n}-A_{2}$, and $p^{n-i-2}$ $\left(A_{1} \cdot A_{2}\right)=0$, then $\Gamma^{i}$ does not link the sum $A_{1}+A_{2}$. Moreover, Alexandroff shows $\S$ that if $A_{1}$ and $A_{2}$ are closed subsets of $E_{n}$,

* Fundamenta Mathematicae, vol. 8 (1926), p. 355.

$\dagger$ Obviously several of the results just stated in $\$ 12$ might properly come under this heading.

$\ddagger A$ proof and extension of the Jordan-Brouwer separation theorem, loc. cit., Corollary $W^{i}$.

$\S$ Gestalt und Lage, p. 178. 
and $\Gamma^{i}$ a cycle of $E_{n}-\left(A_{1}+A_{2}\right)$ which links $A_{1}+A_{2}$ but not $A_{1}$ or $A_{2}$, then there exists a complete $(n-i-2)$-cycle in $A_{1} \cdot A_{2}$ which is homologous to zero in both $A_{1}$ and $A_{2}$ but is not homologous to zero in $A_{1} \cdot A_{2}$.

The epitome of this order of ideas is contained in two theorems of Alexandroff, the first related to the theorem just stated. If every cycle $\Gamma^{i} \sim 0$ in both $E_{n}-A_{1}$ and $E_{n}-A_{2}$, then there exists a cycle $K^{i}$ which links $A_{1}+A_{2}$ if and only if there exists an $(n-i-2)$-complete cycle in $A_{1} \cdot A_{2}$ which is homologous to zero in $A_{1}$ and $A_{2}$, but not in $A_{1} \cdot A_{2}$. Just as the classical PhragmenBrouwer theorem follows from the addition theorem of Alexander, so follows from the theorem just stated the following generalization of the Phragmen-Brouwer theorem. If neither of two closed sets $A_{1}, A_{2}$ separates $E_{n}$, then their sum separates $E_{n}$ if and only if there exists an $(n-2)$-complete cycle in $A_{1} \cdot A_{2}$ which is homologous to zero in both $A_{1}$ and $A_{2}$, but not in $A_{1} \cdot A_{2}$. Thus we have a necessary and sufficient condition that the sum of two closed sets, neither of which separates space, may have a sum that separates space. In this connection it is interesting to note that Kline, in the concluding remarks of his 1927 lecture in New York, gave special mention to the problem of finding necessary and sufficient conditions that the sum of two closed connected sets, neither of which separates three-space, may have a $:$ um that separates three-space. A special case of Alexandroff's theorem is of course a solution of this problem.

I think that sufficient has already been said to indicate the importance of addition theorems in problems concerning the separation, or, in more general terms, the linking, of closed point sets in $E_{n}$. Theorems which concern only the addition of two sets seem to have been sufficient for the treatment of a large number of problems. It is natural, however, to expect that the extension of these investigations to cases of more than two sets may prove valuable, and I find that $\mathrm{E}$. Čech has recently considered this matter. His results* involve too many details to allow of their inclusion here, but he has obtained, for instance, conditions sufficient that a cycle $\Gamma^{i}$ complementary to all of $k$ sets, $A_{1}, A_{2}, \cdots, A_{k}$, and not linking any $k-1$ of these sets, should not link their sum (as a matter of fact, Čech considers

* E. Čech, Trois théorèmes sur l'homologie, Publications de la Faculté des Sciences de 1'Université Masaryk, 1931, Čis. 144. 
the more general case of $s$ cycles, where $s$ is a positive integer, and the condition required is that a certain non-vacuous sum of these shall not link the sum of the given sets).

14. Imbedding Problems. (1) When, in general, can a space $R$ be imbedded in a euclidean space? (2) Under what conditions can a set be imbedded in a Jordan continuum?

The results that have been obtained in the past few years have, I believe, enhanced considerably the importance of both euclidean spaces and Jordan continua. In general, we say that a space $R$ can be imbedded in a space $S$, when there exists a subset $R^{\prime}$ of $S$ whose neighborhoods are defined by its overlappings with neighborhoods of $S$, and such that $R$ and $R^{\prime}$ are homeomorphic. For example, every $n$-dimensional compact metric space can be imbedded in a euclidean space (see below), so that the study of such spaces is identical with the study of bounded closed subsets of euclidean space; in addition, if it is of positive dimension, such a space can be imbedded in a Jordan continuum of the same dimension.

(1) Of central importance among the general topological spaces are the metric separable and the compact metric spaces. That the degree of generality in these conceptions is not as great as might at first be supposed was made evident by Urysohn's discovery that every separable metric space is imbeddable in the Hilbert parallelotope.* That a separable metric space of finite dimension is imbeddable in a compact metric space of the same dimension was proved by Hurewicz. $\dagger$

That every compact metric space of $n$ dimensions can be imbedded in $E_{2 n+1}$ was shown by Menger $\ddagger$ and in combination with the result of the preceding paragraph we have a similar theorem for a separable metric space. More recent proofs of the Menger imbedding theorem have been given by Nöbeling, Lefschetz, and by Pontrjagin and Tolstowa. $\S$

* P. Urysohn, Der Hilbertsche Raum als Urbild der metrischen Räume, Mathematische Annalen, vol. 92 (1924), pp. 302-304.

† Hurewicz's first proof was apparently unsatisfactory-see Menger, Dimensionstheorie, remarks bottom p. 294; however, see W. Hurewicz, Über Einbettung separabler Räume in gleichdimensionale kompakte Räume, Monatshefte für Mathematik und Physik, vol. 37 (1930), pp. 199-208.

$\ddagger$ See Dimensionstheorie, p. 295.

$\S \mathrm{G}$. Nöbeling, Über eine $n$-dimensionale Universalmenge im $R_{2 n+1}$, Mathematische Annalen, vol. 104 (1930), pp. 71-80, S. Lefschetz, On compact spaces, 
In his book, Menger sketched a proof of the theorem that every $n$-dimensional separable metric space is homeomorphic with a subset of $E_{2 n+1}$, and asserted* that there exists for every $n$ a universal $n$-dimensional separable metric space. The proof of this assertion has been provided by Lefschetz and Nöbeling. $\dagger$ Lefschetz first obtains a universal compact metric space by employing the product of a certain series of $(2 n+1)$-dimensional simplexes in $E_{2 n+1}$, and then applying the imbedding theorem for separable spaces. Nöbeling shows that the set of all points of $E_{2 n+1}$ with at most $n$ rational coordinates is an $n$-dimensional universal separable metric space, thus obtaining an existence proof for the universal space and a proof of the imbedding theorem for separable spaces to euclidean spaces in one.

Regarding further results concerning the existence of universal imbedding spaces, special results concerning possibility of imbedding in $E_{2}$, etc., space limitations prevent my going into detail. There remain many unsolved problems concerning imbedding in $E_{2}$ and $E_{3}$, in particular. $\ddagger$

(2) The complete answer to the second question proposed above has been given by Alexandroff and Tumarkin who showed that every compact metric space of positive dimension can be imbedded in a Jordan continuum of the same dimension. $\S$

15. Imbedding by Extension. Given a set of points $M$ in a space $S$, when can it be imbedded by extension in a set $N$ of a given type $T$; that is, when does there exist a set of points $K$ in $S-M$ such that $M+K$ is of type $T$ ?

Annals of Mathematics, vol. 32 (1931), pp. 521-538, L. Pontrjagin and G. Tolstowa, Beweis des Mengerschen Einbettungssatzes, Mathematische Annalen, vol. 105, pp. 734-745.

* Dimensionstheorie, p. 314.

$\dagger$ Loc. cit.

$\ddagger$ Conditions necessary and sufficient that a separable metric space be imbeddable in $E_{1}$ have been given by $\mathrm{L}$. W. Cohen, $A$ characterization of those subsets of metric separable space which are homeomorphic with subsets of the linear continuum, Fundamenta Mathematicae, vol. 14 (1929), pp. 281-303.

$\S \mathrm{P}$. Alexandroff and L. Tumarkin, Beweis des Satzes, dass jede abgeschlossene Menge positiver Dimension in einem lokal zusammenhängenden Kontinuum von derselben Dimension topologisch enthalten ist, Fundamenta Mathematicae, vol. 11 (1928), pp. 141-144. For the zero-dimensional case see W. Sierpinski, Sur les ensembles connexes et non connexes, Fundamenta Mathematicae, vol. 2 (1921), pp. 81-95, and Cohen, loc. cit. 
If $M$ is a closed, compact, totally disconnected point set, $S$ is $E_{n}$, and $N$ is an $H_{n-1}$, then the imbedding by extension is always possible, as shown by Antoine.*

For the case where $S$ is $E_{n}$ and $N$ is an arc, the problem has been solved by Moore and Kline $\nmid(n=2)$ and E. W. Miller $\ddagger$ $(n>2)$, by the furnishing of necessary and sufficient conditions for $M$. For the case where $S$ is $E_{n}$ and $N$ is a Jordan continuum, Gehman showed $\S$ that every bounded continuum $M$ in $E_{2}$ is a subset of a Jordan continuum obtained by adding to $M$ a denumerable infinity of arcs, and Whyburn and Ayres\| extended this result to the case where $S$ is a Jordan continuum. In case $S$ is any open connected subset of a Jordan continuum, and $M$ is a compact closed subset of $S$, I have shown that there exists in $S$ a Jordan continuum containing $M$. $\uparrow$ For the case where $S$ is any metric space, I have shown** that in order that a compact closed subset of $S$ should be a subset of a Jordan contınuum obtained by adding a denumerable infinity of arcs of $S$ to $M$, it is necessary and sufficient that $M$ should be arcwise connected through $S$ and arcwise connected im kleinen through $S$, thus bringing the problem for the case where $N$ is a Jordan continuum and $S$ any metric space to its completion. Due to the nature of the problem (adjustment of a local property, that is,

* L. Antoine, Sur les ensembles parfaits partout discontinus, Comptes Rendus, vol. 173 (1921), pp. 284-285. See also, by the same author, Sur l'homéomorphie de deux figures et de leurs voisinages, Journal de Mathématiques, vol. 4 (1921), pp. 221-325, and Sur les voisinages de deux figures homéomorphes, Fundamenta Mathematicae, vol. 5 (1924), pp. 265-287. Also S. Saks, Sur l'homéomorphie des variétês à deux dimensions, Fundamenta Mathematicae, vol. 5 (1924), pp. 288-320.

$\dagger$ R. L. Moore and J. R. Kline, On the most general plane closed point-set through which it is possible to pass a simple continuous arc, Annals of Mathematics, vol. 20 (1919), pp. 218-223.

$\ddagger$ E. W. Miller, On subsets of a continuous curve which lie on an arc of the continuous curve, American Journal of Mathematics, vol. 54 (1932), pp. 397416.

$\S$ H. M. Gehman, Concerning the subsets of a plane continuous curve, Annals of Mathematics, vol. 27 (1925), pp. 29-46, Theorem 2.

$\|$ G. T. Whyburn and W. L. Ayres, On continuous curves in $n$ dimensions, this Bulletin, vol. 34 (1928), pp. 349-360, Theorem 1.

I R. L. Wilder, On connected and regular point sets, this Bulletin, vol. 34 (1928), pp. 649-655, Theorem 5.

** R. L. Wilder, On the imbedding of subsets of a metric space in Jordan continua, Fundamenta Mathematicae, vol. 19 (1932), pp. 45-64. 
local connectedness), these results have all been obtained by the set-theoretic method.*

It need hardly be indicated that there are many open problems concerning this matter of imbedding by extension. Thus, in $E_{n}$ every arc is a subset of a simple closed curve (because of the accessibility of its end points). However, it is easy to show that not every 2-cell imbedded in $E_{3}$ is a subset of a 2-sphere imbedded in $E_{3} . \dagger$ It might be interesting to determine conditions under which such an extension of a 2-cell may be carried out. Probably the deformability of cycles of the space complementary to the 2-cell, as well as the higher connectivity of the complementary space, must be taken into account here. $\ddagger$

16. Dimension Theory. This theory, in general considered a special province of the set-theoretic topology, has been treated by Menger in his book and by others in several reports. $\$$ That it is, however, amenable to combinatorial attack, has been forecast by several results. To quote Lefschetz,\| "The unavoidable link between dimensionality and combinatorial topology is the Lebesgue-Urysohn-Menger theorem on the order of covering sets." According to an early result of Alexandroff, $\uparrow$ the dimen-

* Imbedding theorems of a more special type have been given by C. M. Cleveland, On the existence of acyclic curves satisfying certain conditions with respect to a given continuous curve, Transactions of this Society, vol. 33 (1931), pp. 958-978, and Zippin, Generalization of a theorem due to C. M. Cleveland, American Journal of Mathematics, vol. 54 (1932), pp. 176-184. Also see W. Stepanoff and W. Tumarkin, Über eine Erweiterung abgeschlossenen Mengen zu Jordanschen Kontinuen derselben Dimension, Fundamenta Mathematicae, vol. 12 (1928), pp. 43-46, and G. T. Whyburn, On the set of all cut points of a continuous curve, ibid., vol. 15 (1930), pp. 185-194.

$\dagger$ By the method used by Alexander (An example of a simply connected surface . . . loc. cit.). Thus, let $T$ be a square plus its interior, and begin attaching the surfaces $S_{i}$ of Alexander at two distinct non-overlapping circular regions of $T$.

$\ddagger$ The same remark applies to the problem of extending the homeomorphism between two simple closed surfaces to the entire space. See end of $\S$.

$\S$ For recent results, G. Nöbeling, Die neuesten Ergebnisse der Dimensionstheorie, Jahresbericht der Deutschen Mathematiker-Vereinigung, vol. 41 (1931), pp. 1-17.

\| S. Lefschetz, On compact spaces, loc. cit.

I P. Alexandroff, Sur la dimension des ensemblesfermés, Comptes Rendus de l'Académie des Sciences, vol. 183 (1926), pp. 640-642. See also references to Brouwer therein. 
sion of a closed set $F$ is the smallest number $m$ having the property that for every $\epsilon>0, F$ is $\epsilon$-deformable into a complex of dimension $m$, thus relating the dimensionality of closed point sets to the dimensionality of ordinary complexes.

Just as in the case of the theory of measure, in setting up a theory of dimension one looks for a theory which will accomplish certain objects intuitively demanded, such as, for instance, that $E_{n}$ shall be, according to the theory proposed, $n$-dimensional. This leads to the main problems of the dimension theory, namely, the setting up of various Rechtfertigungssätze. In the Menger-Urysohn theory most of the desired theo$r$ : ms of this type were established early in the development of the theory, excepting in the case of the question as to whether every $(n-1)$-dimensional closed subset of $E_{n}$ separates some domain, and the question as to whether the product theorem holds. The former question has recently been answered affirmatively by Frankl, Pontrjagin and Alexandroff,* and since, as shown by Urysohn, no closed subset of $E_{n}$ of lower dimension separates any domain, we now have a characterization of $(n-1)$-dimensionality of closed sets in $E_{n}$ by their external properties alone. This raises the question as to whether the same cannot be established for lower dimensionalities. Since the characterizing of $(n-1)$-dimensionality of a set $F$ in $E_{n}$ is accomplished by its separation properties, we should expect that the characterization of lower dimensionalities might be achieved by the linking properties of sets-we shall see below that this is exactly the case. That the product theorem $\dagger$ does not hold for the MengerUrysohn dimension theory has been established lately by Pontrjagin, $\ddagger$ who has demonstrated the existence, in $E_{4}$, of two closed sets $F^{\prime}$ and $F^{\prime \prime}$ such that $\operatorname{dim} F^{\prime}=\operatorname{dim} F^{\prime \prime}=2$, while $\operatorname{dim}$ $\left(F^{\prime} \times F^{\prime \prime}\right)=3$. And here the question arises, can we not set up a dimension theory in which the product theorem holds, without sacrificing any of the other Rechtfertigungssätze?

* F. Frankl and L. Pontrjagin, Ein Knotensatz mit Anwendung auf die Dimensionstheorie, Mathematische Annalen, vol. 102 (1930), pp. 785-789;F. Frankl, Charakterisierung der ( $n-1)$-dimensionalen abgeschlossenen Mengen des $R^{n}$, ibid., vol. 103 (1931), pp. 784-787; P. Alexandroff, Analyse géométrique de la dimension des ensembles fermés, Comptes Rendus, vol. 191 (1930), pp. 475-477.

$\dagger$ That is, $\operatorname{dim}\left(F_{1} \times F_{2}\right)=\operatorname{dim} F_{1}+\operatorname{dim} F_{2}$.

$\ddagger$ L. Pontrjagin, Sur une hypothèse fondamentale de la théorie de la dimension, Comptes Rendus, vol. 190 (1930), pp. 1105-1107. 
The answer to the question, provided by a use of the theory of complete cycles from the standpoint of a unified analysis situs, will appear shortly in a memoir of Alexandroff.* I shall not go in to complete details of Alexandroff's work, but will give enough of its characteristics and results to indicate the general direction in which it proceeds. A set $F$ (hereafter $F$ will always denote a closed set of points) is called $r$-dimensional if it has an essential $(r-1)$-dimensional complete cycle which is homologous to zero in $F$, but no essential complete cycle of higher dimension which is homologous to zero in $F$. According to the modulus used in the homologies, one of course defines thus the dimension modulo $m$, conveniently denoted by $\Delta^{m}(F)$. That this definition agrees with the ordinary intuitive notion of dimension is apparent from a consideration of euclidean elements. If one allows the modulus employed in the definition of $r_{k}$, where $r=\left(r_{1}, r_{2}\right.$, $\left.\cdots, r_{k}, \cdots\right)$ is the cycle employed in this definition, to vary with $k$, we get a complete cycle according to variable modulus, and a corresponding dimension according to variable modulus, which we denote by $\Delta(F)$; this turns out to be identical with the ordinary Brouwer-Menger-Urysohn dimension of closed sets, which is thus a special case of the modular dimensions.

For each of these modular dimensions a dimension theory may be developed. In each such theory we have the summation theorem: "An $n$-dimensional set $F$ is not the sum of countably many $(n-1)$-dimensional closed sets"; the Brouwer invariance principle: "An $n$-dimensional $F$ cannot be carried into a set of lower dimension by optionally small continuous deformations"; the theorem that "Every $n$-dimensional $F$ contains an $n$-dimensional cantorian manifold"; and so on.

Since these dimension theories appear to parallel one another, may they not all be identical in the sense that they all yield the same dimension number for a given set $F$ ? It turns out that in $E_{3}$ they do yield the same dimension but in higher dimensions this is no longer the case, as has been shown by Pontrjagin. $\dagger$

As to the Rechtfertigungssatz referred to above: One of the most interesting results I find in this work is the strong "all-

* Dimensionstheorie. Ein Beitrag zur Geometrie der abgeschlossenen Mengen, Mathematische Annalen, vol. 106 (1932), pp. 161-238; also earlier papers in Comptes Rendus referred to therein.

† Sur une hypothèse $\cdots$, loc. cit. 
gemeine dimensions-theoretische Rechtfertigungssatz". In $E_{n}$, if $\Delta(F)\left(\Delta^{m}(F), m \geqq 2\right)=r$, then there exist spherical neighborhoods $U_{n}$ of $E_{n}$ in which there lie $(n-r-1)$-dimensional polyhedral cycles (cycles modulo $m$ ) which are linked with $F$ in $U_{n}$; if, on the other hand, $\Delta(F)\left(\Delta^{m}(F)\right)$ is smaller than $r$, then every $(n-r-1)$-dimensional polyhedral cycle (cycle modulo $m$ ) complementary to $F$ and lying in a spherical neighborhood $U_{n}$, bounds a complex in $U_{n}-F \cdot U_{n}$. Thus, in $E_{n}$, we are able, by the external properties of a closed set, to determine its dimension, either Menger-Urysohn or modulo $m(m \geqq 2)$.

For the product theorem, we have the interesting result found by Pontrjagin, that if $m$ is zero or a prime number, the dimension modulo $m$ satisfies the product theorem.

It appears, then, that the unified method not only makes an essential contribution to the theory of dimension, but opens up a much wider horizon here just as it does in the other aspects of point set theory discussed above. To quote from Alexandroff's introductory paragraphs:* "The actual application of the combinatorial notion of the homology to the general settheoretic forms permits us to conclude that the dimension theory is not at all an isolated theory, but merely the first section of a general investigation, still in its infancy, of the bounding- and cut- (especially linking-) constructions in closed sets, and as such is to be developed . . . The isolated position of the dimension theory was caused by the lack of a proper foundation in its set-theoretic development; this foundation, which will be given in the present work, indicates . . the end of the self-sufficient existence of a theory and its entrance into a more universal chapter of mathematics." The challenge in these words is clear-cut; it remains only to be seen if it is heeded.

17. Concluding Remarks. I make no claim to the absolute completeness of the above report so far as the totality of results on higher dimensional point sets is concerned; thus, I have included nothing on the researches of Lefschetz on the fixed point problem, the work of Hopf in extending the theory of the Abbildungsgrad of Brouwer; to do all that might be done would necessitate more range than the scope of a symposium lecture allows. I hope, however, that enough has been said to

* Dimensionstheorie, loc. cit. 
convince topologists that the true direction to the investigation of point sets in higher dimensions is indicated by the unified method, which takes into account not only the set-theoretic properties but the connectivity of the point sets as expressed in the Betti numbers. Although, as I have stated above, it is perhaps possible, without reference to the classical combinatorial topology, so to expand the set-theoretic method as to take account of this higher connectivity, I see no reason why we should not consider the theory of complete cycles as that expansion of the set-theoretic method which the set-theorists hope to find; what expression of the character of the higher connectivity can we desire, other than that expressed by connectivity numbers already known?

We have seen, in the above report, that many of the theorems of the set-theoretic topology concerning the separation of space are only special cases of the linking of combinatorial cycles of the complement of a point set. To ignore this fact is to struggle on blindly without a true perspective. And to attempt to build up a theory of higher dimensional point sets without taking into account higher connectivity is to refuse to explore the wider horizon that beckons beyond.

I consider the present situation of a division into two "schools" as the greatest menace to the future development of topology, a division which fosters a lack of true perspective, unscientific animosity, and an unnecessary delay in the progress of our investigations of the structure of space. If what I have said, even if it does not meet universal agreement, leads topologists to face the facts of the situation and hence to some remedy, be it that recommended here or some other, I shall be well satisfied. I hope I have presented evidence enough that the unified method (1) permits us to extend results to higher dimensions, where the properties in the large become complicated, (2) allows great generalization, even in the case of theorems which to the classical set-theoretic point of view appear incapable of generalization, and (3) opens up a vaster field of investigation. I hope that the opportunities for research, indeed, the necessity for research, in this new chapter of analysis situs, will no longer be neglected by the majority of topologists.

The University of Michigan 